\title{
A MODULAR NEUROCONTROLLER FOR A SENSOR-DRIVEN REACTIVE BEHAVIOR OF BIOLOGICALLY INSPIRED WALKING MACHINES
}

\author{
Poramate Manoonpong ${ }^{1,2)}$, Frank Pasemann ${ }^{1)}$, Hubert Roth ${ }^{3)}$ \\ 1) Fraunhofer Institut für Autonome Intelligente Systeme (AIS), Sankt Augustin, Germany \\ frank.pasemann@ais.fraunhofer.de,http://www.ais.fraunhofer.de/INDY \\ 2) Bernstein Center for Computational Neuroscience (BCCN), Göttingen, Germany \\ poramate@nld.ds.mpg.de, http://www.chaos.gwdg.de/ poramate \\ 3) Institut für Regelungs- und Steuerungstechnik (RST), Siegen, Germany \\ hubert.roth@uni-siegen.de, http://www.uni-siegen.de/rst
}

\begin{abstract}
In this article, a modular neurocontroller is presented. It has the capability to generate a reactive behavior of walking machines. The neurocontroller is formed on the basis of a modular structure. It consists of the three different functionality modules: neural preprocessing, a neural oscillator network and velocity regulating networks. Neural preprocessing is for sensory signal processing. The neural oscillator network, based on a central pattern generator, generates the rhythmic movement for basic locomotion of the walking machines while the velocity regulating networks change the walking directions of the machines with respect to the sensory inputs. As a result, this neurocontroller enables the machines to explore in- and out-door environments by avoiding obstacles and escaping from corners or deadlock situations. It was firstly developed and tested on a physical simulation environment, and then was successfully transferred to the six-legged walking machine AMOS-WDO6.
\end{abstract}

Keywords: Walking machines, recurrent neural networks, locomotion control, autonomous robots, modular neural control, obstacle avoidance, sensor-driven reactive behavior, neural oscillator network, central pattern generators.

\section{INTRODUCTION}

The idea behind this article is to investigate the neural mechanisms controlling biologically inspired walking machines represented as sensor-driven systems. The systems are designed in a way that they can react to real environmental stimuli (positive or negative tropism) as they sense without concern for task planning algorithm or memory capacities.

Research in the domain of biologically inspired walking machines has been ongoing for over 10 years. Most of them has been focused on the construction of such machines [1-4] on a dynamic gait control $[5,6]$, and on the generation of an advanced locomotion control [7-9], for instance on rough terrain [10-14]. In general, these walking machines were solely designed for the purpose of motion without the sensing of environmental stimuli.

However, from this research area, only few have presented physical walking machines reacting to an environmental stimulus using different approaches [15-18]. On the one hand, this shows that less attention has been paid to the walking machines performing a reactive behavior. On the other hand, such complex systems can serve as a methodology for study embodied systems consisting of sensors and actuators for explicit agent-environment interactions or they can work as artificial perceptionaction systems.

Here, the biologically inspired six-legged walking machine AMOS-WD06 ${ }^{1}$ is employed as an experimental device for the development and testing of a neurocontroller causing a sensor-driven reactive behavior. This neurocontroller is created on the basis of a modular structure; i.e. it is flexible to adapt for controlling in different walking machines [19] and it is even able to modify for generating different reactive behaviors, e.g. sound tropism (positive tropism) [20]. In this article, it is constructed in the way that it enables the walking machine to avoid the obstacles (negative tropism) by changing the rhythmic leg movements of the thoracic joints. Furthermore, it also prevents the walking machine from getting stuck in corners or deadlock situations by applying hysteresis effects provided by the recurrent neural network of the neural preprocessing module. The following section describes the technical specifications of the walking machine together with its physical simulator. Section 3

\footnotetext{
${ }^{1}$ Advanced MObility Sensor driven-Walking Device 06.
} 
explains a modular neurocontroller together with the subnetworks (modules) for a reactive obstacleavoidance behavior. The experiments and results are discussed in section 4. Conclusions and an outlook on future research are given in the last section.

\section{THE BIOLOGICALLY INSPIRED WALKING MACHINE AMOS-WD06}

The AMOS-WD06 [21] consists of six identical legs and each leg has three joints (three degrees of freedom (DOF)) which is somewhat similar to a cockroach leg [22]. The upper joint of the legs, called thoracic joint, can move the leg forward and backward while the middle and lower joints, called basal and distal joints respectively, are used for elevation and depression or even for extension and flexion of the leg. The levers which are attached to distal joints were built with proportional to the dimension of the machine. And, they are kept short to avoid greater torque in the actuators. This leg configuration provides the machine with the possibility to perform omnidirectional walking; i.e. the machine can walk forward, backward, lateral and turn with different radii. Additionally, the machine can also perform a diagonal forward or backward motion to the left or the right by activating the forward or backward motion together with the lateral left or right motion. The high mobility of the legs enables the walking machine to walk over an obstacle, stand in an upside-down position or even climb over obstacles ${ }^{2}$ (see Fig. 1).

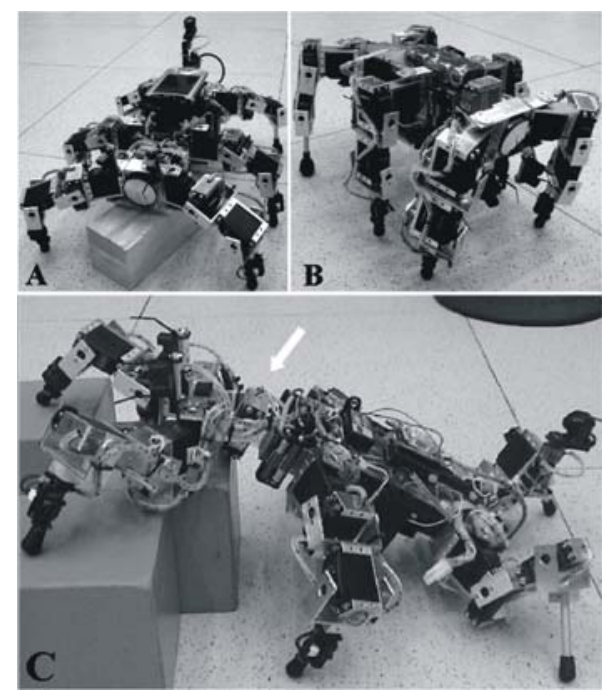

Fig. 1 - (A) The AMOS-WD06 walks over an obstacle with the maximum height of $7 \mathrm{~cm}$, (B) stands in an upside-down position and $(C)$ climbs over obstacles with the help of an active backbone joint (arrow).

\footnotetext{
${ }^{2}$ Note that the controller driving the machine also with the backbone joint is not described in this paper; it will be published elsewhere together with the technical details for climbing.
}

Inspired by invertebrate morphology of the american cockroach's trunk and its motion (see Fig. 2), a backbone joint which can rotate in a horizontal axis was constructed. It is desired to imitate like a connection between the first (T1) and second (T2) thoracic of a cockroach. Thus, it will provide enough movement for the machine to climb over an obstacle by rearing the front legs up to reach the top of an obstacle and then bending them downward during step climbing (compare Fig. 1C).

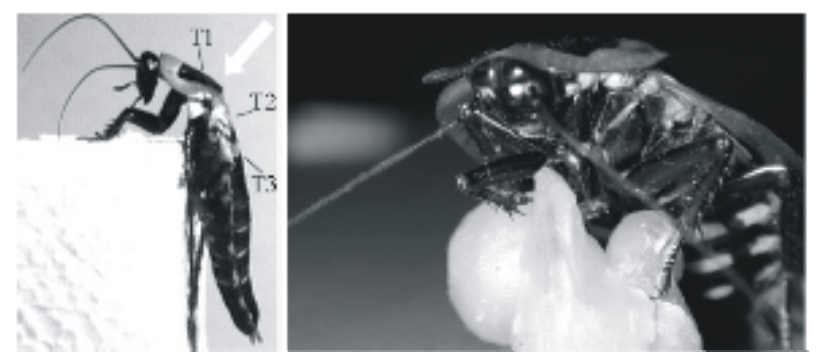

Fig. 2 - A cockroach climbs over large obstacles. It can bend its trunk downward at the joint between the

first (T1) and second (T2) thoracic to keep the legs

close to the top surface of the obstacles for an

optimum climbing position and even to prevent unstable actions (modified from R.E. Ritzmann 2004 [22] with permission).

However, this active backbone joint will not be activated in a normal walking condition of the machine. Mainly, it is used to connect the trunk (second thoracic), where two middle legs and two hind legs are attached, with the head (first thoracic), where two forelegs are installed. The trunk and the head are formed with the maximum symmetry to keep the machine balanced for the stability of walking. They are also designed to be as narrow as possible to ensure optimal torque from the supporting legs to the center line of the trunk. Moreover, a tail with two DOF rotating in the horizontal and vertical axes was implemented on the back of the trunk. In fact, this actively moveable tail which can be manually controlled is used only to install a mini wireless camera for monitoring the environment while the machine is walking. But the tail also gives the walking machine a more animallike appearance, e.g. in analogy to a scorpion's tail with a sting [23].

All leg joints are driven by analog servomotors producing a torque between 80 and $100 \mathrm{Ncm}$. The backbone joint is driven by a digital servomotor with a torque between 200 and $220 \mathrm{Ncm}$. For the tail joints, micro-analog servomotors with a torque around $20 \mathrm{Ncm}$ were selected. The height of the walking machine is $12 \mathrm{~cm}$ without its tail and the weight of the fully equipped robot (including 21 servomotors, all electronic components and a mobile processor) is approximately $4.2 \mathrm{~kg}$. In addition, the walking machine has six Infra-Red (IR) sensors. 
Two of them, which can detect the obstacle at a long distance between $20-150 \mathrm{~cm}$, were fixated at the forehead while the rest of them, operating at a shorter distance between $4-30 \mathrm{~cm}$, were fixated at the two forelegs and two middle legs. They help the walking machine to detect obstacles and prevent its legs from hitting obstacles, like chair or desk legs. Also, one upside-down detector was implemented beside the machine trunk. It provides the information of upside-down position of the walking machine.

All in all the AMOS-WD06 has 21 active degrees of freedom, 7 sensors and 1 wireless camera, and therefore it can serve as a reasonably complex platform for experiments concerning the functioning of a neural sensor-driven system. However, to test neurocontrollers and to observe the reactive behavior of the walking machine (e.g. obstacle avoidance), it was firstly done through a physical simulation environment, namely "Yet Another Robot Simulator" (YARS). The simulator is based on Open Dynamics Engine (ODE) [24]. It provides a defined set of geometries, joints, motors and sensors which is adequate to create the AMOS-WD06 with IR sensors in a virtual environment with obstacles. The basic features of the simulated walking machine are closely coupled to the physical walking machine, e.g. weight, dimension, motor torque and so on. It consists of body parts (head, backbone joint, trunk and limbs), servomotors, IR sensors and an additional tail. The simulated walking machine with its virtual environment is shown in Fig. 3.
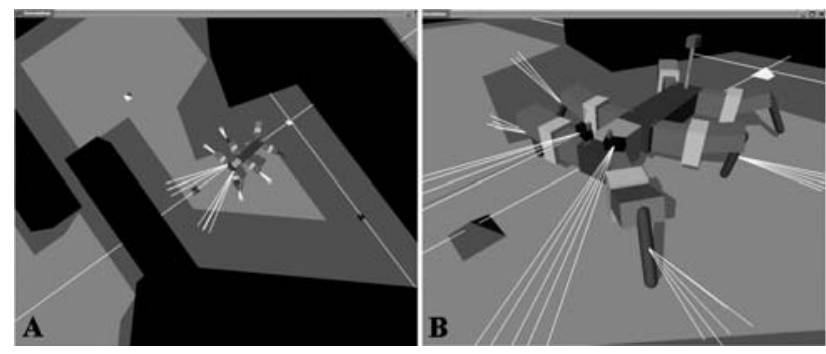

Fig. 3 - Different views of the simulated walking machine in its environment.

Furthermore, the simulator enables an implementation, which is faster than real time and which is precise enough to present the corresponding behavior of the physical walking machine. This simulation environment is also connected to the Integrated Structure Evolution Environment (ISEE) [25] which is a software platform for developing neurocontrollers. In the final stage, a developed neurocontroller after the test on the simulator is then applied to the physical walking machine to

\footnotetext{
${ }^{3}$ http://www.ais.fraunhofer.de/INDY/, see menu item TOOLS.
}

demonstrate the behavior in the real environment. The controller is then programmed into a mobile processor (a personal digital assistant (PDA)) with an update frequency of up to $75 \mathrm{~Hz}$. The PDA is interfaced with the Multi-Servo IO-Board (MBoard $)^{4}$ which digitizes sensory input signals and generates a pulse width modulation (PWM) signal at a period of $20 \mathrm{~ms}$ to command the servomotors. The communication between the PDA and the MBoard is accomplished via an RS232 interface at $57.6 \mathrm{kBits}$ per second.

\section{NEURAL PERCEPTION-ACTION SYSTEMS}

In order to create the robust and effective neurocontroller which is able to generate exploration and reactive obstacle avoidance behaviors, the dynamical properties of recurrent neural networks are utilized. The standard additive neuron model with sigmoidal transfer function together with its time-discrete dynamics is given by:

$a_{i}(t+1)=B_{i}+\sum_{j=1}^{n} W_{i j} \tanh \left(a_{j}(t)\right) \quad i=1, \ldots, n$,

where $n$ denotes the number of units, $a_{i}$ their activity, $B_{i}$ represents a fixed internal bias term together with a stationary input to neuron $i$, and $W_{i j}$ synaptic strength of the connection from neuron $j$ to neuron $i$. The output of neurons is given by the sigmoid $o_{i}=\tanh \left(a_{i}\right)$. Input units are configured as linear buffers.

The modular neurocontroller for the desired behaviors are divided into three subnetworks (modules) which are the signal processing network, the neural oscillator network and the velocity regulating network. All networks are described in detail in the following sections.

\subsection{SIGNAL PROCESSING NETWORK}

The perception systems are driven by using six IR sensors. The minimal recurrent controller (MRC) structure [26] is applied for preprocessing IR signals. This controller has been originally developed for controlling a miniature Khepera robot [27], which is a two wheeled platform. Here, it is modified for controlling the walking directions of the machine to avoid obstacles or escape from a corner and even a deadlock situation.

To do so, all signals of IR sensors (IR1, IR2, IR3, IR4, IR5 and IR6) are mapped onto the interval $[-\mathbf{1},+\mathbf{1}]$, with $\mathbf{- 1}$ representing "no obstacles", and +1

\footnotetext{
${ }^{4}$ http://www.ais.fraunhofer.de/BE/volksbot/mboardcontent.html.
} 
"an obstacle is detected". Then, the three sensory signals on each side (right or left) are simply combined in a linear domain of the sigmoid transfer function at hidden neurons; i.e. each of them is multiplied with a small weight, here $W_{1,2,3,4,5,6}=$ 0.15 . The output signals of the hidden neurons are directly connected to the output neurons (Out1, Out2) while the final output signals of the network (Output1, Output2) will be connected to another network called the velocity regulating networks (VRNs) described later. The parameters of the preprocessing network were manually adjusted on the basis of its well understood functionality [26].

First, the bias term $(B)$ of the hidden neurons is set to determine a threshold value of the sum of the sensory inputs, e.g. 0.2. When the measured value is greater than the threshold in any of the three sensory signals, excitation of the hidden neuron on the corresponding side occurs. Consequently, the activation of each hidden neuron can vary in the range between $\approx-0.245$ ("no obstacles is detected") and $\approx 0.572$ ("all three sensors on the appropriate side simultaneously detect obstacles").

Furthermore, the weights from the hidden to the output units are set to a high value, i.e. $W_{7,8}=25.0$, to eliminate the noise of the sensory signals. In fact, these high multiplicative weights drive the signals to switch between two saturation domains, one low $(\approx-1)$ and the other high $(\approx+1)$. After that, the selfconnection weights $\left(W_{9,10}\right)$ of Out1 and Out2 were manually adjusted to derive a reasonable hysteresis input interval which results to an appropriate turning angle of the walking machine when the obstacles are detected. Hereby, they are set to 4.0. Finally, the recurrent connections $\left(W_{11,12}\right)$ between output neurons were symmetrized and manually adjusted to the value of -2.5 . This guarantees the optimal functionality described later. The resulting network is shown in Fig. 4.

The set-up parameters cause that the network can eliminate the noise of the sensory signals. The network can even determine the turning angle in accordance with the width of the hysteresis loop; i.e. the wider the loop, the larger the turning angle is. The capability of the network in filtering the sensory noise together with the hysteresis loop of the network are shown in Fig. 5.

In addition, there is a third hysteresis phenomenon involved which is associated to the even loop [28] between the two output neurons. In general conditions, only one neuron at a time is able to get a positive output, while the other one has a negative output, and vice versa. The phenomenon is presented in Fig. 6.

By applying the described phenomena, the walking machine is able to avoid the obstacles and escape from corners as well as deadlock situations.

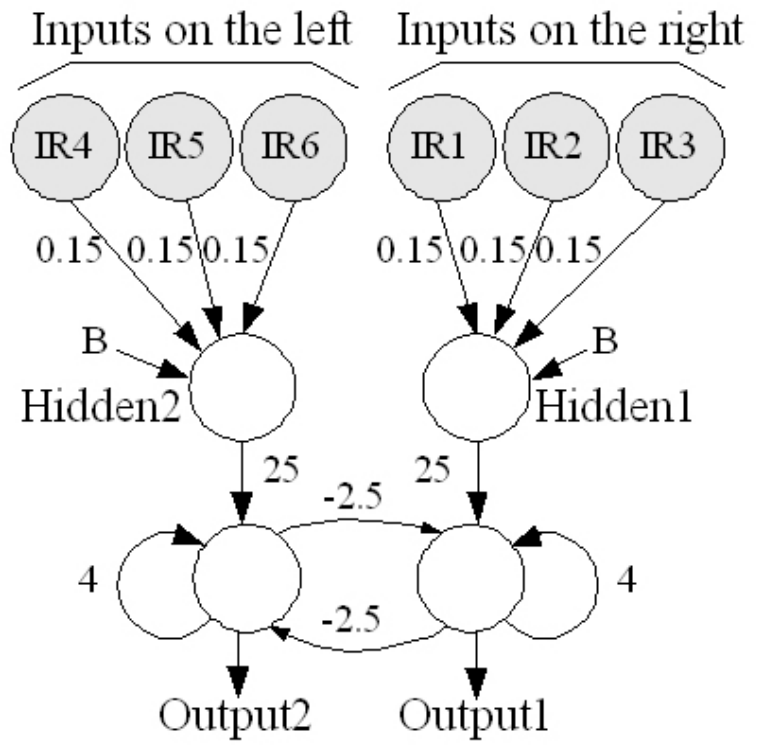

Fig. 4 - The signal processing network of six IR sensors with the appropriate weights for controlling the walking direction of the machine to avoid obstacles and to prevent the machine from getting stuck in corners or deadlock situations.
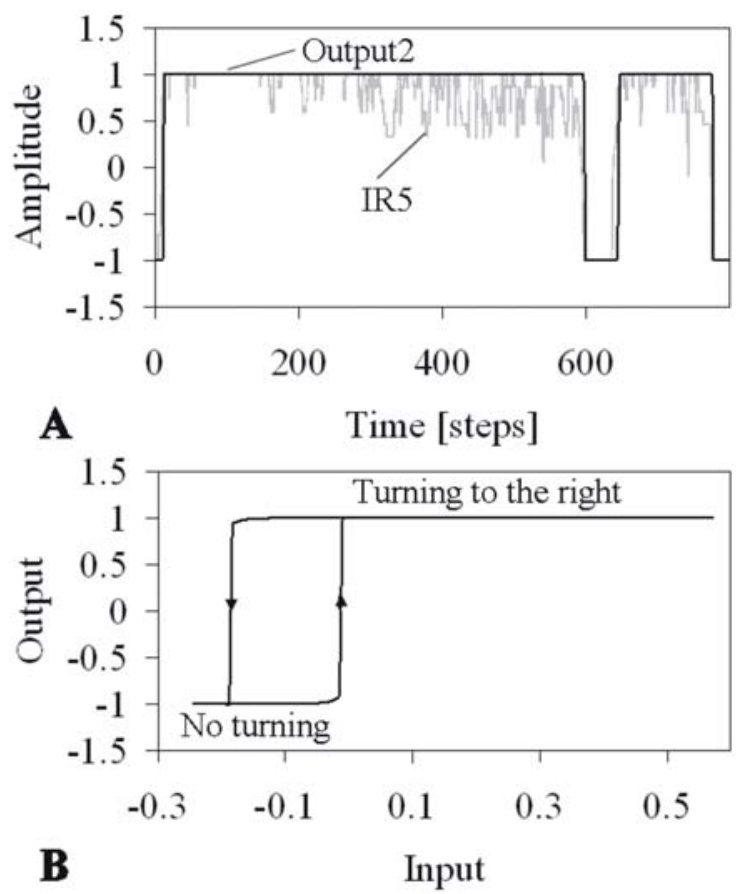

Fig. 5 - (A) The sensory signal (IR5, gray line) before preprocessing and the output signal (Output2, solid line) after preprocessing. (B) The "hysteresis effects" between input and output signals of Out2. In this situation, the input of Out 2 varies between $\approx \mathbf{- 0 . 2 4 5}$ and $\approx 0.572$ back and forth while the input of Out1 is set to $\approx \mathbf{- 0 . 2 4 5}$ ("no obstacles are on the right side").

Here, when the output of Out 2 is active $(\approx 1)$; i.e.

"obstacles are on the left side", then the walking machine will be driven to turn right until the output becomes inactivated $(\approx-1)$. On the other hand, if such condition occurs for Out1, the input of Out1 will derive the same hysteresis effect as the input of Out 2 does. 


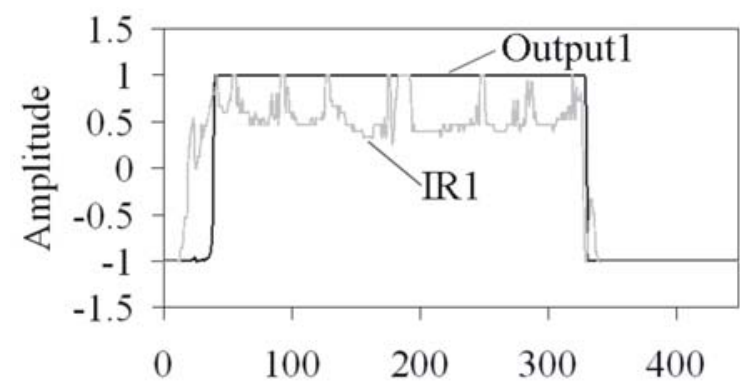

$\mathbf{A}$

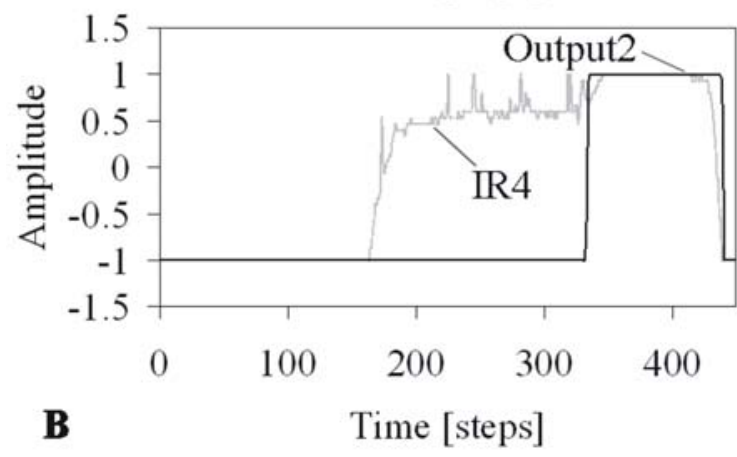

Fig. 6 - (A) and (B) present the sensory signals (IR1 and IR4, gray line) and the output signals (Output1 and Output2, solid line), respectively. Due to the inhibitory synapses between two output neurons and the high activity of Out1 (A), Output2 (B) is still inactive although IR4 becomes activated at around 170 time steps. At around 320 time steps, the switching condition between Output1 and Output2 occurs because IR1 becomes inactivated, meaning "no obstacles detected" while IR4 is still active, meaning "obstacles detected".

Finally, Output1 and Output2 of the preprocessing network together with the velocity regulating networks, described below, control and switch the behavior of the walking machine; for instance, switching the behavior from "walking forward" to "turning left" when there are obstacles on the right, or vice versa. The network outputs also determine in which direction the walking machine should turn in corners or deadlock situations depending on which sensor side has been previously active. In special situations, like walking toward a wall, both sides (right and left) of IR sensors might get positive outputs at the same time, and, because of the velocity regulating networks, the walking machine is able to walk backward. During walking backward, the activation of the sensory signal of one side might be still active while the other might be inactive. Correspondingly, the walking machine will turn into the opposite direction of the active signal and it can finally leave from the wall.

\subsection{NEURAL OSCILLATOR NETWORK}

The concept of neural oscillators for walking machines has been studied in various works [29-33]. For instance, Kimura et al. [34] presented a neural oscillator network with four neurons. The network has been used to control a four-legged robot TEKKEN where each neuron of the network drives each hip joint of the legs.

Here a so-called "2-neuron network" [35] is employed. It is used as a central pattern generator (CPG) $[36,37]$ which corresponds to one basic principle of locomotion control of walking animals $[38,39]$. The network consists of two neurons (see Fig. 7A). It has already been implemented successfully on other walking machines [19, 40]. The same weight matrixes presented there are used here. Consequently, it generates the oscillating output signals corresponding to a quasi-periodic attractor (see Fig. 7B). They are used to drive the motors directly for generating the appropriate locomotion of the AMOS-WD06.

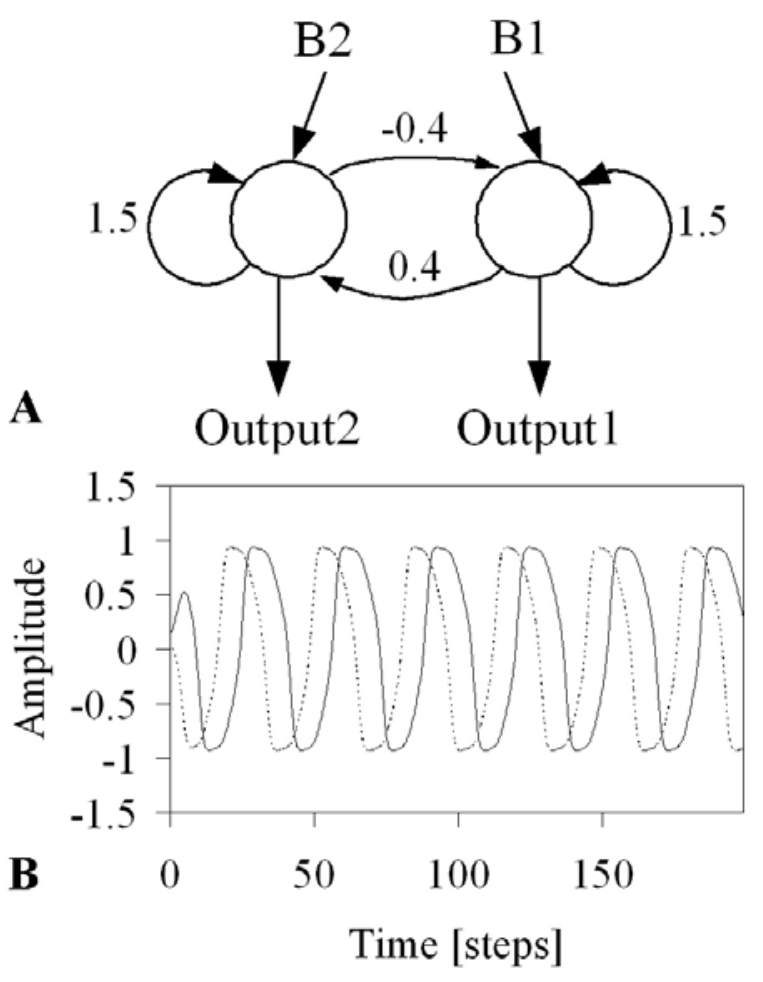

Fig. 7 - (A) The structure of the 2-neuron network with the synaptic weights for the purpose. $B 1$ and $B 2$ are bias terms with $B 1=B 2=0.01$. (B) The output signals of neurons 1 (dashed line) and 2 (solid line) from the neural oscillator network. The output of neuron 1 is used to drive all thoracic joints and the active backbone joint while the output of neuron 2 is used to drive all basal and all distal joints.

This network is implemented on a PDA with an update frequency of $25.6 \mathrm{~Hz}$. It generates a sinusoidal output with a frequency of approximately $0.8 \mathrm{~Hz}$. By using asymmetric connections from the oscillator outputs to corresponding motor neurons, a typical tripod gait for the six-legged walking machine is obtained (see Fig. 8). This typical gait enables an efficient motion, where the diagonal legs are paired and move together. 

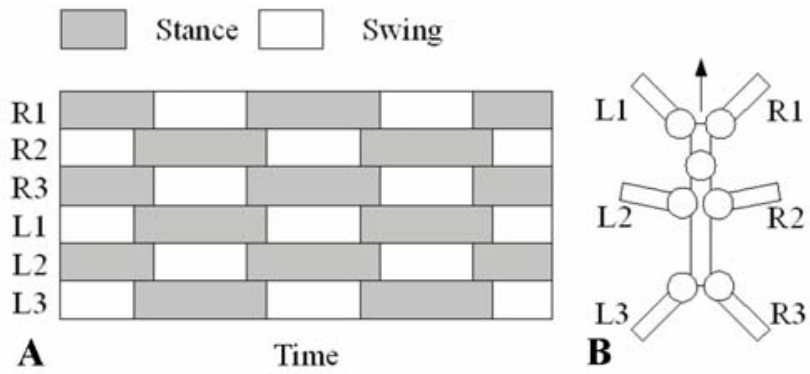

Fig. 8 - (A) The typical tripod gait. X-axis represents time and $y$-axis represents the legs. During the swing phase (white blocks) the feet have no ground contact. During the stance phase (gray blocks) the feet touch the ground. (B) The orientation of the legs of the AMOS-WD06

\subsection{THE VELOCITY REGULATING NETWORK}

To change the motions, e.g. from walking forward to backward and to turning left and right, the simplest way is to perform a 180 degree phase shift of the sinusoidal signals which drive the thoracic joints. To do so, the velocity regulating network (VRN) is introduced. The network used is taken from [41]. It performs an approximate multiplication-like function of two input values $x, y$ $\in[-\mathbf{1},+\mathbf{1}]$. For this purpose the input $x$ is the oscillating signal coming from the neural oscillator network to generate the locomotion and the input $y$ is the sensory signal coming from the neural preprocessing network to drive the reactive behavior. The output signal of the network will be used to drive the thoracic joints. Fig. 9A presents the network consisting of four hidden neurons and one output neuron. Fig. 9B shows that the output signal which gets a phase shift of 180 degrees, when the sensory signal (input $y$ ) changes from -1 to +1 .

\subsection{THE MODULAR NEUROCONTROLLER}

The combination of all three networks (modules) leads to an effective neural network for reactive behavior control in changing environments. On the one hand, one oscillating output signal from the neural oscillator network is directly connected to all basal and distal joints. On the other hand, the other output is connected to the thoracic joints only indirectly, passing through all hidden neurons of the VRNs through the so called $x$-inputs. The outputs of the signal processing network are also connected to all hidden neurons of the VRNs as $y$-inputs. Thus, the rhythmic leg movement is generated by the neural oscillator network and the steering capability of the walking machine is realized by the VRNs in accordance with the outputs of the signal processing

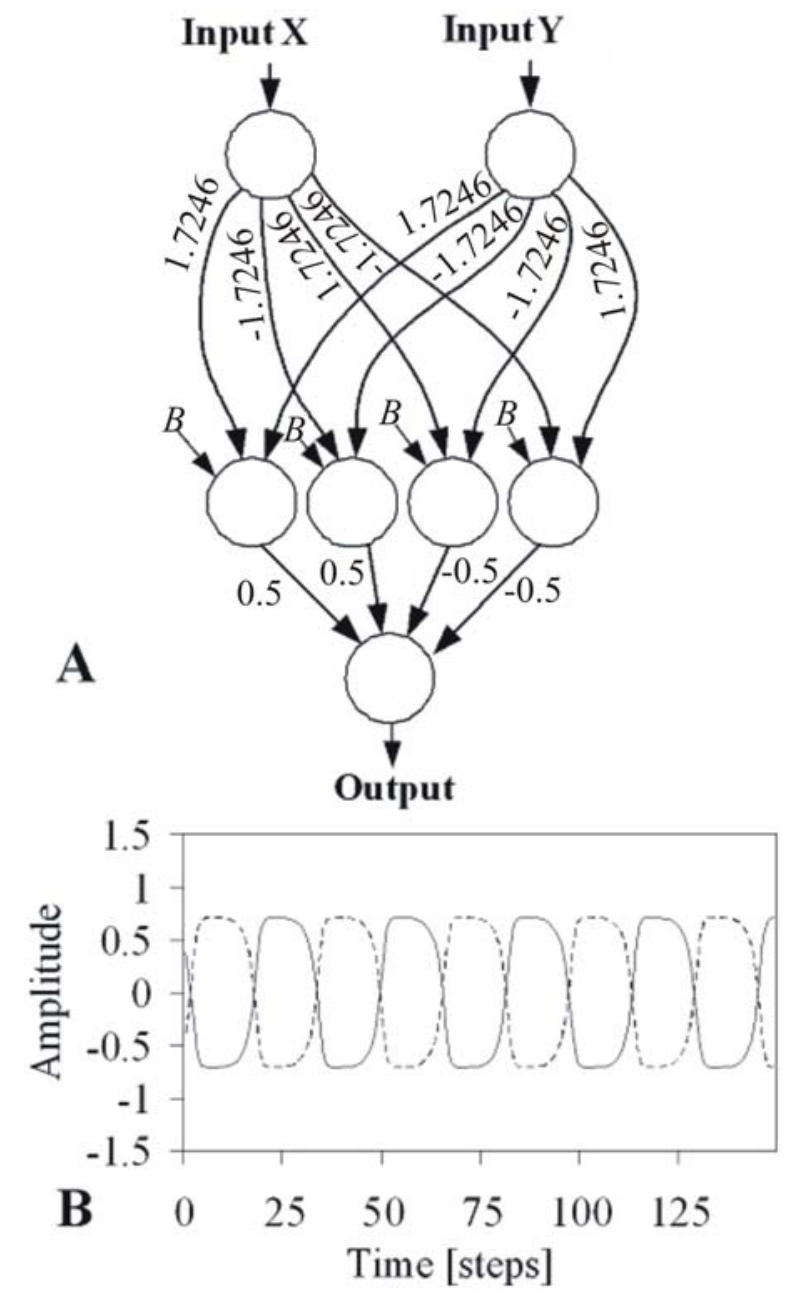

Fig. 9 - (A) The VRN with four hidden neurons and the given bias terms $B$ which are all equal to $\mathbf{- 2 . 4 8 2 8 5}$. The Input $x$ is the oscillating signal coming from the neural oscillator and the Input $y$ is the output signal of the neural preprocessing. (B) The output signal (solid

line) when the input $y$ is equal to +1 and the output signal (dashed line) when the input $y$ is equal to -1 .

network. The structure of this controller and the location of the corresponding motor neurons on the walking machine AMOS-WD06 are shown in Fig. 10.

\section{EXPERIMENTS AND RESULTS}

The performance of the modular neural network shown in Fig. 10 is firstly tested on the physical simulation with a complex environment (see Fig. 3) and then it is downloaded into the mobile processor of the AMOS-WD06 for a test on the physical autonomous robot.

The simulated walking machine and the physical walking machine behave qualitatively. The sensory information of IR sensors is used to modify the machine behavior as expected from a perceptionaction system. If the obstacles are presented on either the right or the left side, the controller will change the rhythmic movement at the thoracic joints of the legs, causing the walking machine to turn on 


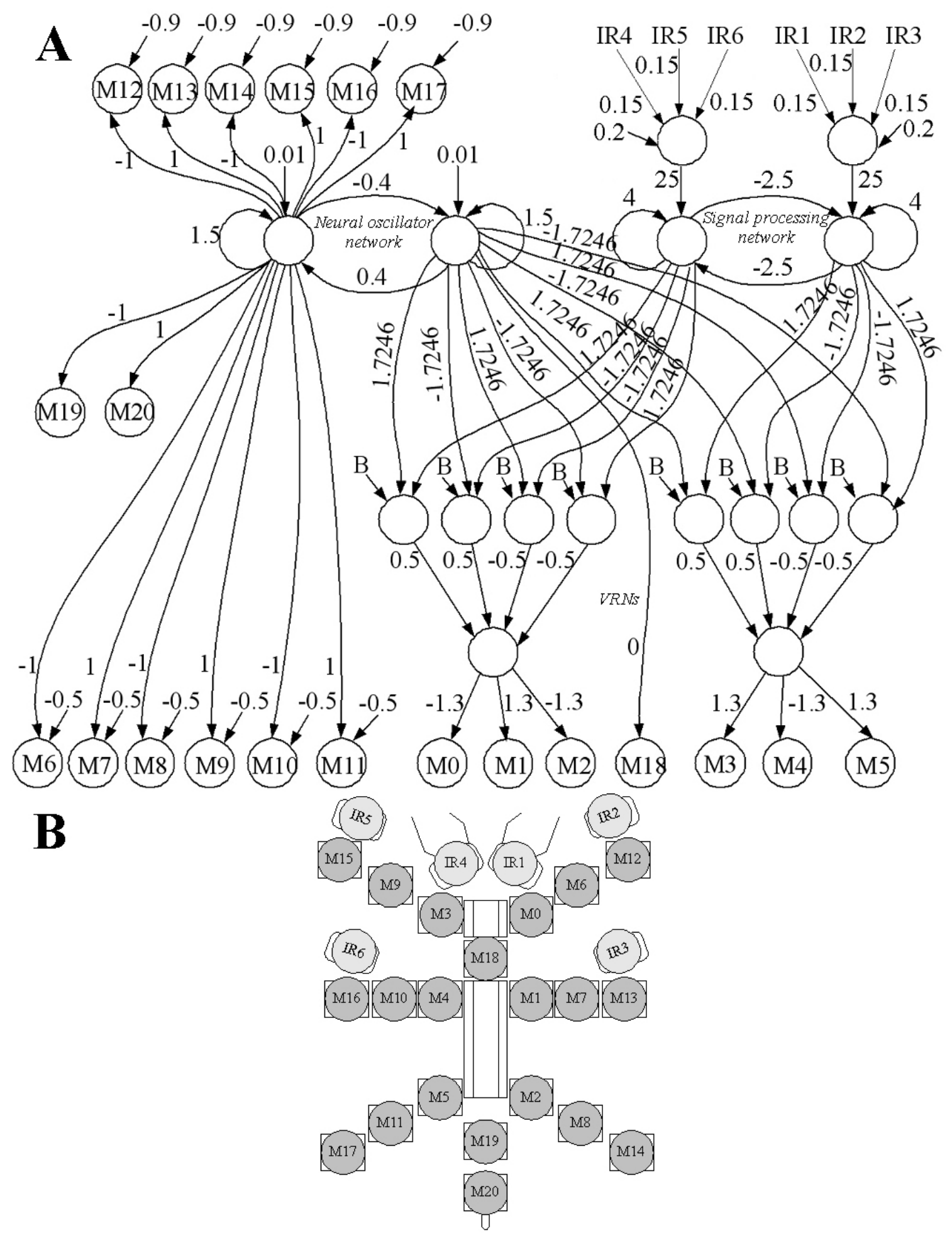

Fig. 10 - (A) The modular neurocontroller. It generates a tripod gait which is modified when obstacles are detected. The bias terms $(B)$ of the VRNs are again all equal to $\mathbf{- 2 . 4 8 2 8 5}$. Six IR sensors are directly connected to the input neurons of the signal processing network. If the obstacle is detected, the outputs of the signal processing make the walking machine turn because the VRNs change the quasi-periodic signals at the thoracic joints. (B) The layout of all motor and input neurons of the AMOS-WD06.

the spot and immediately avoiding the obstacles. In some situations, like approaching a corner or a deadlock situation, the preprocessing network determines the turning direction. The modification of the motor neurons with respect to the sensory inputs is exemplified in Fig. 11.
MO, M1 and M2 of the thoracic joints (compare Fig. 11, left) are turned into the opposite direction because one of the left sensors (here, IR4) detects the obstacle. On the other hand, M3, M4 and M5 of the thoracic joints are turned into the opposite direction when, at least, one of the right sensors 

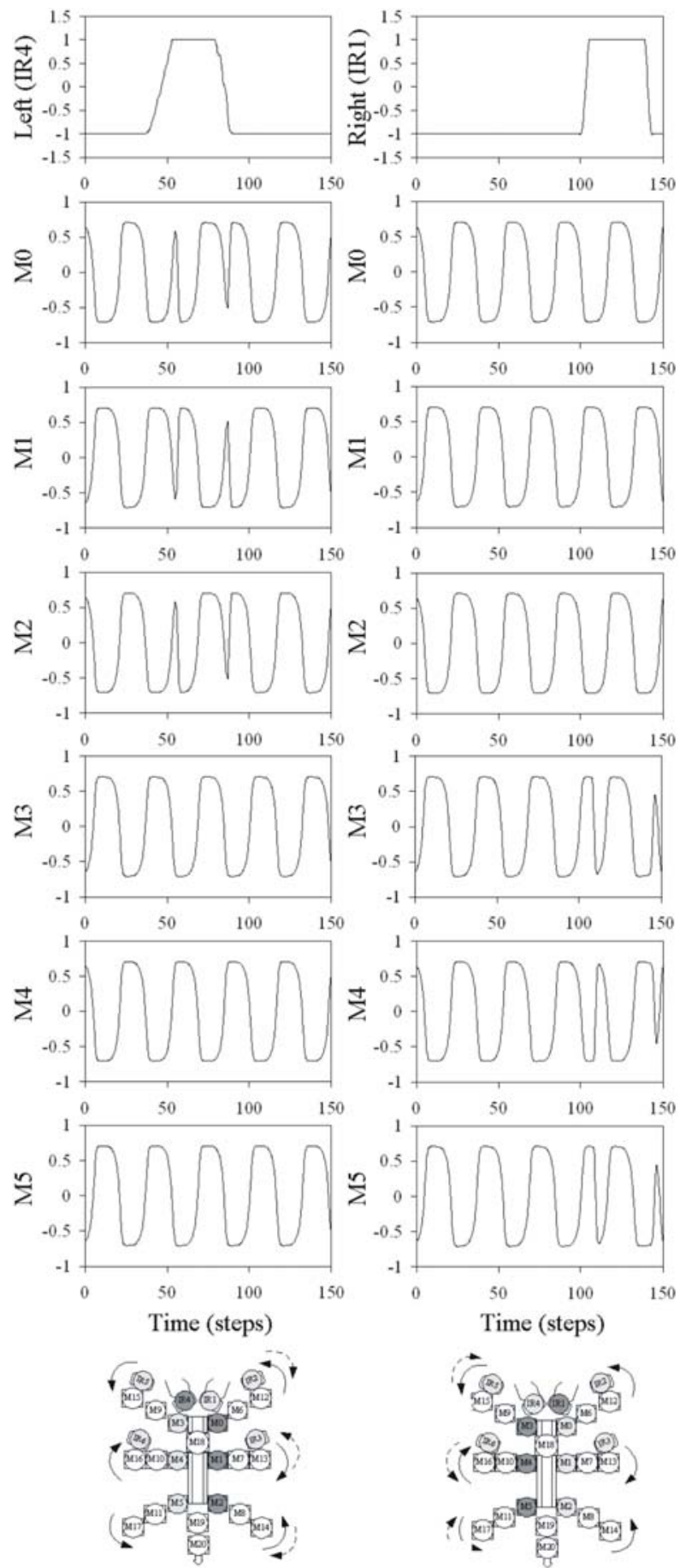

Fig. 11 - Left: The left sensor (IR4) detected the obstacle while the other sensors (IR1, IR2, IR3, IR5 and IR6) did not detect the obstacle; this caused motor neurons (MO, M1 and $M 2)$ on its right to change into

the opposite direction. As a result, the walking machine will turn right. Right: If the obstacle is detected at the right of the walking machine (here, it was detected by only IR1), then the motor neurons (M3, M4 and M5) on its left are reversed.

Consequently, the walking machine will turn left.

(here, IR1) is active (compare Fig. 11, right). In special situations, e.g. walking toward a wall or detecting obstacles on both sides, IR sensors of both sides may be active simultaneously. Thus, $M 0, M 1$, M2, M3, M4 and M5 of the thoracic joints are reversed which causes the walking machine to walk backward and eventually it is able to leave the wall. Fig. 12 presents the example reactive behavior of the walking machine driven by the sensory inputs together with the modular neurocontroller. A series of photos on the left column in Fig. 12 shows that the walking machine can escape from a deadlock situation and it can also protect its legs from colliding with the legs of a chair (see middle column in Fig. 12). Moreover, it was even able to turn away from the unknown obstacles which were sensed by the sensors at the forehead and then were detected by the sensors on the left legs (see right column in Fig. 12). More demonstrations of reactive behaviors of the AMOS-WD06 are referred to video clips at, www.chaos.gwdg.de/ poramate/AMOSWD06.html.

As demonstrated, the modular neurocontroller is adequate to successfully complete the obstacle avoidance task. Due to the capability of the controller, the walking machine can perform an exploration task (wandering behavior) without getting stuck in the corner or the deadlock-like situation.

\section{CONCLUSION}

The six-legged walking machine AMOS-WD06 is presented as a reasonably complex robot platform to test a neurocontroller generating the robust sensor-driven exploration and obstacle avoidance behaviors. The modular neurocontroller was designed as a neural network consisting of a signal processing network for preprocessing IR signals, a neural oscillator network for generating basic locomotion, and the velocity regulating networks (VRNs) for changing the locomotion appropriately. The controller is used to generate the walking gait of the machine and enable it to perform the reactive behavior; for instance, exploring an in-door environment by wandering around, avoiding obstacles when they are detected, and leaving from a corner-like deadlock situation. The controller has been tested successfully in the physical simulation environment as well as on the real world walking machine. Thus, we were able to reproduce these basic behaviors, generally achieved for wheeled robots, also for a machine with many degrees of freedom. The generated behaviors are of course essential for an autonomous walking machine. More demanding tasks will be related to the use of additional sensors. Therefore, future research we will make use of auditory signals coming from a stereo auditory sensor. It will be used for navigation based on sound tropism. Finally all these different reactive behaviors will be fused into one modular neurocontroller, where modules have to cooperate or compete as in versatile perception-action systems. 


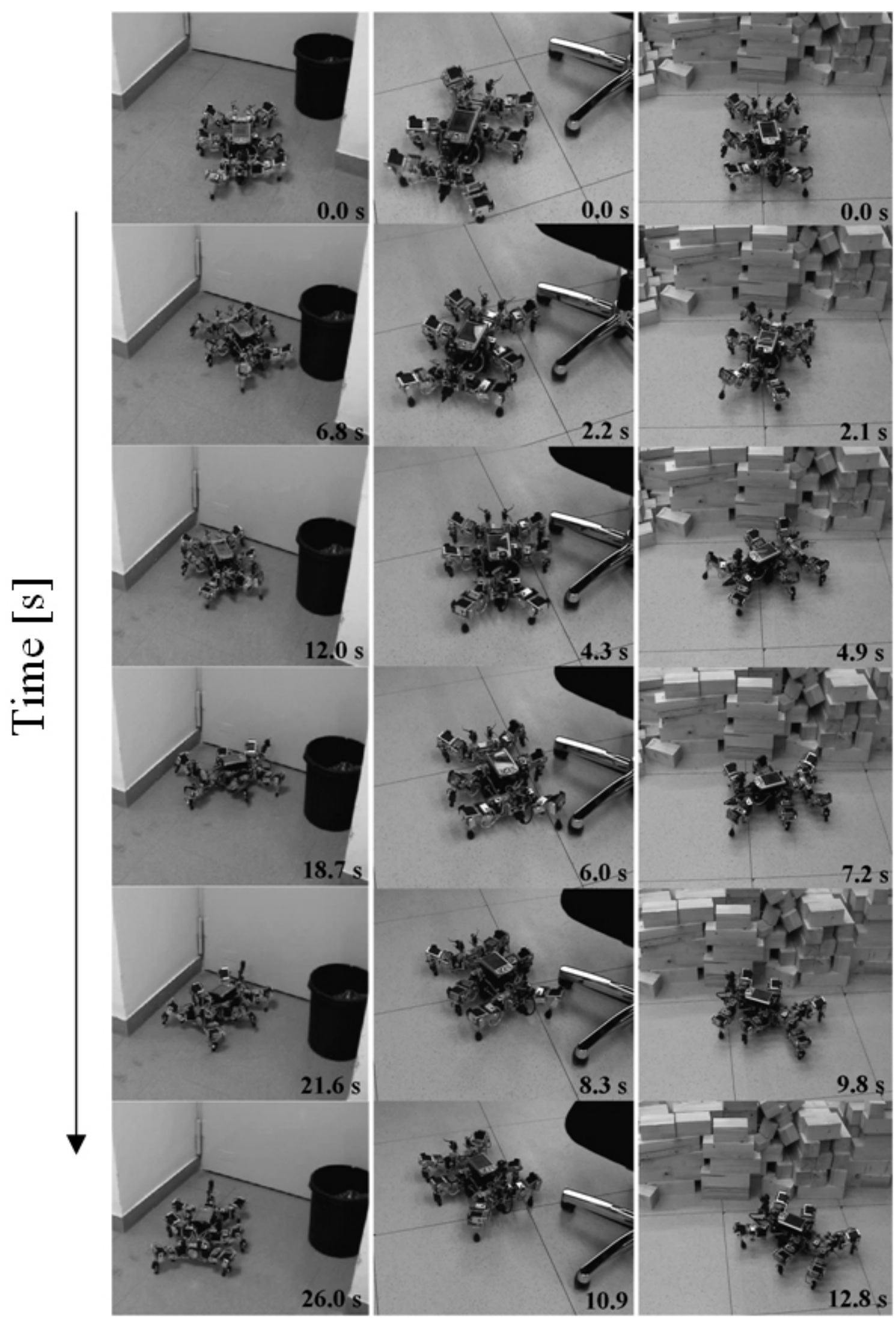

Fig. 12 - The Examples of the behavior driven by the IR sensors of the AMOS-WD06. Left: The AMOS-WD06 escaped from a corner-like deadlock situation without getting stuck. Middle: It was able to protect its legs from colliding with the leg of a chair which was detected by the sensors installed on the right legs. Right: It turned away from the unknown obstacles which was detected by the sensors at the forehead (IR1 and IR4) and then at the left legs (IR5 and IR6). 


\section{ACKNOWLEDGEMENTS}

The Integrated Structure Evolution Environment (ISEE) software platform for the evolution of recurrent neural networks and Yet Another Robot Simulator (YARS) were kindly provided by Keyan Zahedi, Martin Hülse, and Steffen Wischmann from Fraunhofer AIS.

\section{REFERENCES}

[1] J. E. Clark, J. G. Cham, S. A. Bailey, E. M. Froehlich, P. K. Nahata, R. J. Full, and M. R. Cutkosky. Biomimetic Design and Fabrication of a Hexapedal Running Robot. Proceedings of the IEEE International Conference on Robotics and Automation, 2001, pp. 3643-3649.

[2] J. Hilljegerdes, D. Spenneberg, and F. Kirchner. The Construction of the Four Legged Prototype Robot ARAMIES. Proceedings of the International Conference on Climbing and Walking Robots (CLAWAR'05), 2005, pp. 335342.

[3] R. Breithaupt, J. Dahnke, K. Zahedi, J. Hertzberg, and F. Pasemann. Robo-Salamander an approach for the benefit of both robotics and biology. Proceedings of the International Conference on Climbing and Walking Robots (CLAWAR'02), 2002, pp. 55-62.

[4] R. D. Quinn, G. M. Nelson, R. J. Bachmann, D. A. Kingsley, J. Offi, and R. E. Ritzmann. Insect Designs for Improved Robot Mobility. Proceedings of the International Conference on Climbing and Walking Robots (CLAWAR'01), 2001, pp. 69-76.

[5] M. Toyomasu and A. Shinohara. Developing dynamic gaits for four legged robots. Proceedings of the International Symposium on Information Science and Electrical Engineering, 2003, pp. 577-580.

[6] R. Kurazume, K. Yoneda, and S. Hirose. Feedforward and feedback dynamic trot gait control for quadruped walking vehicle. Autonomous Robots 12(2), 2002, pp. 157-172.

[7] C. R. Linder. Self-organization in a Simple Task of Motor Control Based on Spatial Encoding. Adaptive Behavior 13(3), 2005, pp. 189-209.

[8] H. Cruse, V. Dürr, and J. Schmitz. Control of hexapod walking in biological systems. Proceedings of the International Symposium on Adaptive Motion of Animals and Machines, 2003, On CD.

[9] M. A. Jimenez and P. Gonzalez de Santos. Terrain adaptive gait for walking machines. International Journal of Robotics Research 16(3), 1997, pp. 320-339.

[10] D. Spenneberg and F. Kirchner. Embodied
Categorization of Spatial Environments on the basis of Proprioceptive Data. Proceedings of the International Symposium on Adaptive Motion in Animals and Machines, 2005, on CD.

[11] E. Celaya and J. M. Porta. A Control Structure for the Locomotion of a Legged Robot on Difficult Terrain. IEEE Robotics and Automation Magazine 5(2), 1998, pp. 43-51.

[12] J. Albiez, T. Luksch, K. Berns, and R. Dillmann. Reactive Reflex-based control for a four-legged walking machine. Robotics and Autonomous Systems 44(3), 2003, pp. 181-189.

[13] J. Ingvast, C. Ridderström, F. Hardarson, and J. Wikander. Warp1: Towards walking in rough terrain - control of walking. Proceedings of the International Conference on Climbing and Walking Robots (CLAWAR'03), 2003, pp. 197204.

[14] K. S. Espenschied, R. D. Quinn, R. D. Beer, and H. J. Chiel. Biologically based distributed control and local reflexes improve rough terrain locomotion in a hexapod robot. Robotics and Autonomous Systems 18(1-2), 1996, pp. 59-64.

[15] A. D. Horchler, R. E. Reeve, B. Webb, and R. D. Quinn. Robot phonotaxis in the wild: A biologically inspired approach to outdoor sound localization. Advanced Robotics 18(8) (2004), pp. 801-816.

[16] D. Filliat, J. Kodjabachian, and J. A. Meyer. Incremental evolution of neural controllers for navigation in a 6 legged robot. Proceedings of the International Symposium on Artificial Life and Robotics, Oita University Press, 1999, pp. 745-750.

[17] K. S. Ali and R. C. Arkin. Implementing Schema-theoretic Models of Animal Behavior in Robotic Systems. Proceedings of the International Workshop on Advanced Motion Control (AMC’98), Coimbra, Portugal, 1998, pp. 246-253.

[18] R. D. Brooks. A Robot That Walks: Emergent Behavior from a Carefully Evolved Network. Neural Computation 1(2) (1989), pp. 253-262.

[19] P. Manoonpong, F. Pasemann, and J. Fischer. Modular neural control for a reactive behavior of walking machines. Proceedings of the IEEE Symposium on Computational Intelligence in Robotics and Automation (CIRA'05), Helsinki University of Technology, Finland, 2005, pp. 403-408.

[20] P. Manoonpong, F. Pasemann, J. Fischer, and H. Roth. Neural Processing of Auditory Signals and Modular Neural Control for Sound Tropism of Walking Machines. International Journal of Advanced Robotic Systems 2(3), 2005, pp. 223234.

[21] P. Manoonpong and F. Pasemann. Advanced 
MObility Sensor driven-Walking Device 06 (AMOS-WD06). Proceedings of the Third International Symposium on Adaptive Motion in Animals and Machines, Robot data sheet, Ilmenau, 2005, p. R23.

[22] R. E. Ritzmann, R. D. Quinn, and M. S. Fischer. Convergent evolution and locomotion through complex terrain by insects, vertebrates and robots. Arthropod Structure and Development 33(3), 2004, pp. 361-379.

[23] F. T. Abushama. On the behaviour and sensory physiology of the scorpion Leirus quinquestriatus. Animal behaviour 12 (1964), pp. 140-153.

[24] R. Smith. Open Dynamics Engine v0.5 User Guide. http://ode.org/ode-0.5-userguide.html, 2004.

[25] M. Hülse, S. Wischmann, and F. Pasemann. Structure and function of evolved neurocontrollers for autonomous robots. Connection Science 16(4), 2004, pp. 249-266.

[26] M. Hülse and F. Pasemann. Dynamical Neural Schmitt Trigger for Robot Control. Proceedings of the International Conference on Artificial Neural Networks (ICANN 2002), J. R. Dorronsoro (Ed.), LNCS Vol. 2415, 2002, pp. 783-788.

[27] F. Mondada, E. Franzi, and P. Ienne. Mobile robot miniaturisation: A tool for investigation in control algorithms. Proceedings of the Third International Symposium on Experimental Robotics, 1993, pp. 501-513.

[28] F. Pasemann. Discrete dynamics of two neuron networks. Open Systems and Information Dynamics 2, 1993, pp. 49-66.

[29] K. Matsuoka. Mechanisms of frequency and pattern control in the neural rhythm generators. Biolog. Cybern. 56, 1987, pp. 345-353.

[30] W. Ilg, J. Albiez, H. Jedele, K. Berns, and R. Dillmann, Adaptive periodic movement control for the four legged walkingmachine BISAM. Proceedings of the IEEE International Conference on Robotics and Automation, 1999, pp. 2354-2359.

[31] G. Endo, J. Morimoto, J. Nakanishi, and G. Cheng. An Empirical Exploration of a Neural Oscillator for Biped Locomotion Control. Proceedings of the IEEE International Conference on Robotics and Automation, 2004, pp. 3036-3042.

[32] L. Righetti and A. J. Ijspeert. Programmable Central Pattern Generators: an application to biped locomotion control. Proceedings of the IEEE International Conference on Robotics and Automation, 2006, pp. 1585-1590.

[33] I. Markelic. Evolving a neurocontroller for a fast quadrupedal walking behavior. Master thesis, Institut für Computervisualistik Arbeitsgruppe Aktives Sehen, Universität Koblenz-Landau, Germany, 2005.

[34] H. Kimura, Y. Fukuoka, and A. H. Cohen. Biologically Inspired Adaptive Dynamic Walking of a Quadruped Robot. Proceedings of the International Conference on the Simulation of Adaptive Behavior (SAB2004), 2004, pp. 201-210.

[35] F. Pasemann, M. Hild, and K. Zahedi. SO(2)Networks as Neural Oscillators. Proceedings of the International Work- Conference on Artificial and Natural Neural Networks (IWANN 2003), LNCS Vol. 2686, pp. 144-151.

[36] F. Kirchner and D. Spenneberg. A Biologically Inspired Approach towards Autonomous Real World Robots. Complex Systems Science in BioMedicine. Kluwer Academic - Plenum Publishers, New York, 2004 (in Press).

[37] K. Nakada, T. Asai, and Y. Amemiya, Biologically-inspired locomotion controller for a quadruped walking robot: Analog IC implementation of a CPG-based controller. Journal of Robotics and Mechatronics 16(4), 2004, pp. 397-403.

[38] H. Cruse. The functional sense of central oscillations in walking. Biological Cybernetics 86(4), 2002, pp. 271-280.

[39] E. Marder and D. Bucher. Central pattern generators and the control of rhythmic movements. Current Biology 11(23), 2001, pp. 986-996.

[40] J. Fischer, F. Pasemann, and P. Manoonpong. Neuro-Controllers for Walking Machines - an evolutionary approach to robust behavior. Proceedings of the International Conference on Climbing and Walking Robots (CLAWAR'04), 2004, pp. 97-102.

[41] J. Fischer. A Modulatory Learning Rule for Neural Learning and Metalearning in Real World Robots with Many Degrees of Freedom. $\mathrm{Ph}$.D. Thesis, university of Muenster, Germany, Shaker Verlag, 2004.

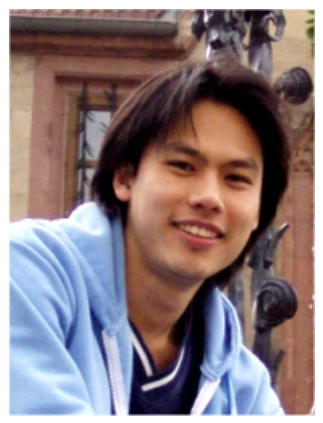

Poramate Manoonpong was born in Nan, Thailand, in 1978. He received a B.Eng. degree in mechanical engineering from the King Mongkut's University of Technology Thonburi, Thailand, in 2000, a M.Sc. degree in mechatronics from Fachhochschule Ravensburg-Weingarten, Germany, in 2002, and a Ph.D. degree in electrical engineering and computer science from the University of Siegen, Germany, in 
2006. As a Ph.D. student, he worked in the areas of robotics, mechatronic systems, biologically inspired walking machines, evolutionary robotics and artificial neural networks at Fraunhofer institute for Autonomous Intelligent Systems (AIS), Sankt Augustin, Germany.

$\mathrm{He}$ is currently a researcher at Bernstein Center for Computational Neuroscience (BCCN) Goettingen, Germany. His research interests include locomotion control of walking machines, dynamics of recurrent neural networks, embodied cognitive systems.

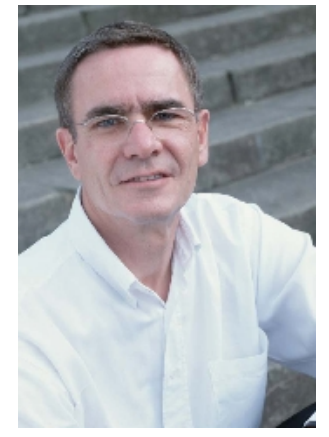

\section{Frank Pasemann}

Studied Physics and Mathematics; Diploma (1971), Dr. rer.nat. (1977) and Habilitation (1985) in Theoretical Physics. Headed research groups at the Technical University Clausthal, Research Centre Jülich (19931996), the Max Planck-Institute for Mathematics in the Sciences, Leipzig (1997-1999), the University Jena (2000-2001), and at the Fraunhofer Institute for Autonomous Intelligent Systems, Sankt Augustin (since 2002). Since 2004 Honor.Prof., Institute of Cognitive Science, University Osnabrück. Main research interests: Dynamics of Recurrent Neural Networks, Evolutionary Robotics, and Cognitive Systems as Complex Adaptive Systems.

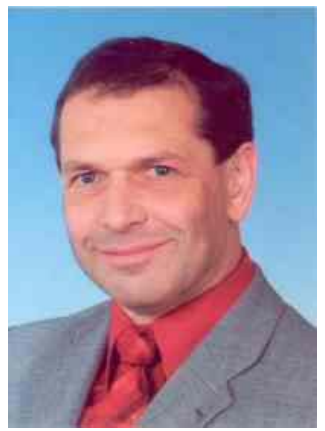

Hubert Roth was studying "Electrical Engineering" at University Karlsruhe, Germany, from 1974 until 1979 with emphasis in "Control Engineering and Computer Science". Until 1984 he was working as a Scientific Co-worker at the Institute for Automatic Control Engineering at the University Karlsruhe and got his doctoral degree in Electrical Engineering. From 1984 until 1988 he was employed as a System Design Engineer at Dornier System, Spacecraft Control Department, in Friedrichshafen, Germany. After this industrial internship he went back to academia and worked as a Professor for Control and Sensor Systems at the University of Applied Sciences, RavensburgWeingarten, Germany, for 12 years. In the year 2000 he became head of the Institute for Automatic Control Engineering in the faculty of Electrical Engineering and Computer Science at the University of Siegen, Germany. In parallel he is Vice Chair of the Centre for Sensor Systems (ZESS) at the University of Siegen and is head of the working group "Automation, Mechatronics and Medical Engineering" in this centre. Prof. Roth is Vice Chair of the Technical Committee on Computers and Telematics of the "International Federation of Automatic Control" (IFAC).

The main teaching interests of Prof. Roth are in the fields of Control Theory, Robotics, Mechatronic Systems and Attitude Control for Space Applications. His research interests are focused on Mobile Robotics, Aero-space applications and Telecontrol as well as environmental exploration. 